\title{
Contrast-Enhanced Mammography: Technique, Indications, and Review of Current Literature
}

\author{
Gemmy Hannsun $^{1} \cdot$ Stephen Saponaro $^{2} \cdot$ Paul Sylvan $^{2} \cdot$ Azadeh Elmi $^{1,2}$ (B)
}

Accepted: 14 October 2021 / Published online: 3 November 2021

(C) The Author(s) 2021

\begin{abstract}
Purpose of Review To provide an update on contrast-enhanced mammography (CEM) regarding current technique and interpretation, the performance of this modality versus conventional breast imaging modalities (mammography, ultrasound, and MRI), existing clinical applications, potential challenges, and pitfalls.

Recent Findings Multiple studies have shown that the lowenergy, non-contrast-enhanced images obtained when performing CEM are non-inferior to full-field digital mammography with the added benefit of recombined postcontrast images, which have been shown to provide comparable information compared to MRI without sacrificing sensitivity and negative predictive values. While CEMs' usefulness for further diagnostic characterization of indeterminate breast findings is apparent, additional studies have provided strong evidence of potential roles in screening intermediate to high-risk populations, evaluation of disease extent, and monitoring response to therapy,
\end{abstract}

This article is part of theTopical collection on Breast Imaging.

Azadeh Elmi

aelmi@health.ucsd.edu

Gemmy Hannsun

ghannsun@health.ucsd.edu

Stephen Saponaro

ssaponaro@eisenhowerhealth.org

Paul Sylvan

psylvan@eisenhowerhealth.org

1 Breast Imaging Division, Department of Radiology, UC San Diego Health, Koman Family Outpatient Pavilion, 9400 Campus Point Dr, La Jolla, San Diego, CA 92037, USA

2 Lucy Curci Cancer Center, Eisenhower Medical Center, 39000 Bob Hope Drive, Rancho Mirage, CA 92270, USA particularly in patients in whom MRI is either unavailable or contraindicated. Others have shown that some patients prefer CEM over MRI given the ease of performance and patient comfort. Additionally, some health systems may find significantly reduced costs compared to MRI. Currently, CEM is hindered by the limited availability of CEM-guided tissue sampling and issues of intravenous contrast administration. However, commercially available CEM-guided biopsy systems are on the horizon, and small changes in practice workflow can be quickly adopted. As of now, MRI remains a mainstay of high-risk screening, evaluation of the extent of disease, and monitoring response to therapy, but smaller studies have suggested that CEM may be equivalent to MRI for these indications, and larger confirmatory studies are needed.

Summary CEM is an emerging problem-solving breast imaging modality that provides complementary information to conventional imaging modalities and may potentially be used in place of MRI for specific indications and/ or patient populations.

Keywords Breast cancer - Contrast-enhanced mammography · Breast MRI - Background parenchymal enhancement $\cdot$ Breast cancer screening

\section{Introduction}

Contrast-enhanced mammography (CEM) is an emerging modality that has the potential to become a first-line problem-solving tool in breast imaging. Although digital mammography has undoubtedly demonstrated its effectiveness as a screening tool with sensitivity for cancer detection ranging from 70 to $78 \%$ [1-3], mammography continues to be limited in the setting of dense breasts with 
sensitivities in the range of 47.8-64.4\% [4]. Additionally, the inherent lack of soft tissue contrast between malignancy and normal surrounding tissue continues to plague breast imagers to this day. The introduction of digital breast tomosynthesis (DBT) has reduced the impact of overlapping breast tissue, improving the conspicuity of masses and architectural distortions. However, DBT does not address the challenge of poor contrast between cancer and normal tissue. The use of dynamic contrast-enhanced breast magnetic resonance imaging (MRI) with gadolinium-based contrast agents reduces the impact of these particular issues, increasing the sensitivity of lesion detection to $92 \%$ (95\% CI $[0.89,0.94])$ in a 2017 metaanalysis [5]. The limitations of MRI include extended imaging times, increased costs, and high rates of false positives [6]. CEM combats these issues by taking advantage of the inherent hypervascularity typically induced by malignancy and applying dual-energy imaging techniques with post-processing software to improve the diagnostic accuracy of this modality to approach that of MRI.

Application of contrast in breast imaging was proposed even before development of digital mammography. Contrast-enhanced mammography has evolved over the past few decades and finally was approved by the U.S. Food and Drug Administration (FDA) in 2011 as an adjunct imaging modality. This technique is performed utilizing digital subtraction angiographic technique, which relied upon algorithmic subtraction of a pre-contrast, masked image from post-contrast angiogram while maintaining the breast in the same position [7]. This concept of temporal subtraction was soon thereafter adapted to digital mammography [8]. After a non-contrast mammogram was obtained, intravenous contrast was administered via a power injector. Multiple post-contrast images of the same breast were obtained, with the breast kept in the same position over a 5to 10-min period. Despite successfully identifying enhancing breast lesions, multiple challenges arose. During image acquisition, the breast compression required to decrease intrinsic scatter from fibroglandular tissue would impede tissue perfusion, contributing to decreased lesion enhancement. Only one breast projection could be practically obtained per bolus, drastically limiting its utility compared to breast MRI with provided 3D data via a single bolus. Lastly, prolonged imaging times lead to patient motion, further decreasing contrast-to-noise. Eventually, a dual-energy or spectral technique was developed, allowing multiple breast projections to be obtained using a single contrast bolus [9], which has become the preferred method.

As CEM provides an assessment of lesion vascularity, it can be surmised that many current applications of this modality would be similar to indications typically requiring breast MRI. CEM could be conceivably used for breast cancer screening, preoperative staging, characterization of indeterminate findings, and monitoring of response to therapy. Recent studies have provided promising data in cancer screening and evaluating treatment response to neoadjuvant therapy, suggesting that CEM may be comparable to MRI in specific populations [10••, 11]. Additionally, increased specificity and PPV have been reported for diagnosing malignancy when CEM is performed in the workup of suspicious findings noted on screening examination [12]. CEM could be offered as a reasonable alternative with comparable diagnostic performance in specific patient populations that cannot obtain MRI.

This review will discuss current CEM technique and interpretation, compare the performance of this modality versus conventional breast imaging modalities (mammography, ultrasound, and MRI), provide a case-based review of common imaging findings and current applications in the literature, as well as analyze common challenges and pitfalls seen in everyday practice. As of this publication, there is no Breast Imaging-Reporting and Data System (BIRADS) lexicon used to interpret CEM. Current interpretation relies on a combination of terms already in use in the BI-RADS lexicon for mammography and MRI. A discussion of potential terms specific for CEM will also be provided in preparation for the upcoming iteration of the BIRADs manual.

\section{Technique}

CEM is often performed with standard mammographic equipment only requiring minimal modifications, typically including specialized software permitting dual-energy technique as well as an additional copper filter allowing for the formation of higher-energy X-rays [13]. Before beginning the examination, patients are screened for a history of allergic-like and physiologic reactions to iodinated contrast per guidelines established by the American College of Radiology [14]. Women of child-bearing age are screened for pregnancy as data of iodinated contrast use during gestation are limited, and other imaging modalities could be preferable. No animal studies have demonstrated teratogenic or mutagenic effects of iodinated contrast administered during pregnancy, and no evidence of teratogenesis in humans has been identified $[15,16]$.

After intravenous access has been established (via an antecubital vein usually using a 20-gauge IV catheter), low-osmolar nonionic iodinated contrast is administered via a power injector set-up at a dose of $1.5 \mathrm{~mL} / \mathrm{kg}$ and a rate of $3 \mathrm{~mL} / \mathrm{s}$. Typically, a 2 min waiting period from the time of contrast administration to image acquisition is recommended based on data from multiple prior studies which have shown that cancer typically becomes visible after $2 \mathrm{~min}$ and less conspicuous by the $8 \mathrm{~min}$ post- 
injection time point, predominantly secondary to physiologic background enhancement and tumor washout $[17,18]$. Jochelson et al. demonstrated that enhancement of cancer with iodinated contrast is relatively time-independent during the first 2-10 min post-injection [10••].

After the brief waiting period, both the low- and highenergy images can be obtained. The lower-energy image is acquired most commonly utilizing a molybdenum filter to produce an energy spectrum ranging from 26 to $32 \mathrm{kVp}$, which best optimizes the visualization of breast tissue and microcalcifications [19]. While maintaining the breast in the same compression, a higher-energy image is obtained using copper-filtered X-rays, increasing the X-ray energy range to around $45-49 \mathrm{kVp}$ [20] to take advantage of the $\mathrm{K}$-edge of iodine $(33.2 \mathrm{keV})$.

The two images are processed using logarithmic subtraction algorithms to provide a recombined image that highlights areas of iodine uptake while simultaneously suppressing normal background tissue. Although iodine has a higher attenuation coefficient compared to breast tissue, the iodine concentration in most lesions after contrast administration is too low to produce an appreciable difference on standard digital mammography. Because the $\mathrm{K}$-edge of iodine occurs in the photon energy range used for digital mammography, the inherent suddenly increased absorption that typically occurs at its K-edge can be used to distinguish lesion enhancement from the non-enhancing background. The dual-energy algorithm uses three inputs to produce the recombined image: the low-energy image, the high-energy image, and the thickness of the compressed breast to provide a gray-scale density map of iodine concentration per pixel. The breast thickness is assumed to be equal in thickness throughout, although uniform compression is typically not feasible, particularly at the edges of the paddles. Because X-ray photons are exponentially attenuated, the data are logarithmically transformed before undergoing weighted subtraction to produce the final recombined image.

Medio-lateral oblique (MLO) and craniocaudal (CC) projections are subsequently obtained per institutional protocol. A review of 84 articles totaling over 14,000 patients has shown that no established acquisition protocol has been determined. In that review, the most common convention performed was MLO projections of each breast followed by $\mathrm{CC}$ projections of each breast (Fig. 1). Of the studies that provided length of acquisition time, a total of 65 of 66 studies reported obtaining all the desired projections within $10 \mathrm{~min}$ of contrast administration [21]. After all the desired images have been obtained, IV access should be maintained for at least 15-30 min while monitoring for signs and symptoms of adverse contrast reactions. The same study by Zanardo et al. reported that of the over 14,000 patients, a total of 30 patients developed an adverse reaction, with only one patient requiring brief ICU admission with following day discharge.

\section{Interpretation}

The interpretation of CME relies on the evaluation of the low-energy and post-processed subtraction images. The high-energy images are typically noninterpretable due to poor soft tissue contrast and are only used to produce postprocessed images. The study can be interpreted on the same dedicated PACS workstations used to interpret mammograms, although different hanging protocols may be required to optimize workflow.

Low-energy images are initially interpreted for the presence of abnormalities, such as calcifications and masses. Despite the presence of iodinated contrast, low-energy images have been shown in the literature to be non-inferior to conventional digital mammography [22•]. Subtraction images are then reviewed for areas of increased contrast enhancement relative to background parenchymal enhancement (BPE).

Understanding the concept of BPE, as in MRI, is critical to successful interpretation. Current descriptors of BPE are adapted from the MRI BI-RADs lexicon, including minimal, mild, moderate, and marked (Fig. 2). A study of over 200 patients has shown significant inter-reader agreement in characterizing background enhancement in CEM and MRI between the readers and the modalities themselves [23]. Despite anecdotal evidence, it has been shown that menstrual cycle timing appears to have minimal impact on the extent of parenchymal enhancement [23, 24].

Abnormal enhancement in CEM is typically characterized as increased contrast uptake relative to enhancing background tissue. Subsequently, as BPE increases in intensity, the likelihood of overlooking a lesion also increases. Regions of focal enhancement should be reevaluated on the low-energy images, as some findings may become more conspicuous retrospectively. The area can also be further evaluated on spot compression or tomosynthesis images. The next step will be a sonographic evaluation of the area. If there is no sonographic or mammographic correlate, MRI should be performed (if feasible). At any of these examinations, if there are any suspicious findings, a biopsy should be performed. If no imaging correlate and in the absence of capability for CEM-guided biopsy, 6-month follow-up CEM should be recommended (Fig. 3).

While many malignant lesions demonstrate avid enhancement, so do many benign entities, ultimately decreasing the specificity of the modality, similar to MRI. Many common benign enhancing breast lesions including fibroadenoma, papilloma, $\mathrm{PASH}$, radial scar, ductal 

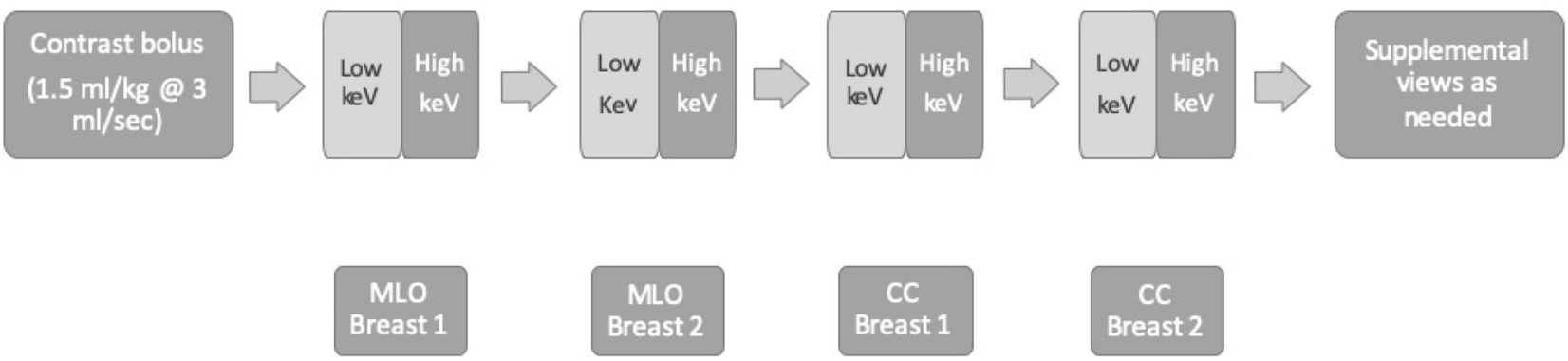

Fig. 1 Standard protocol for performing CEM
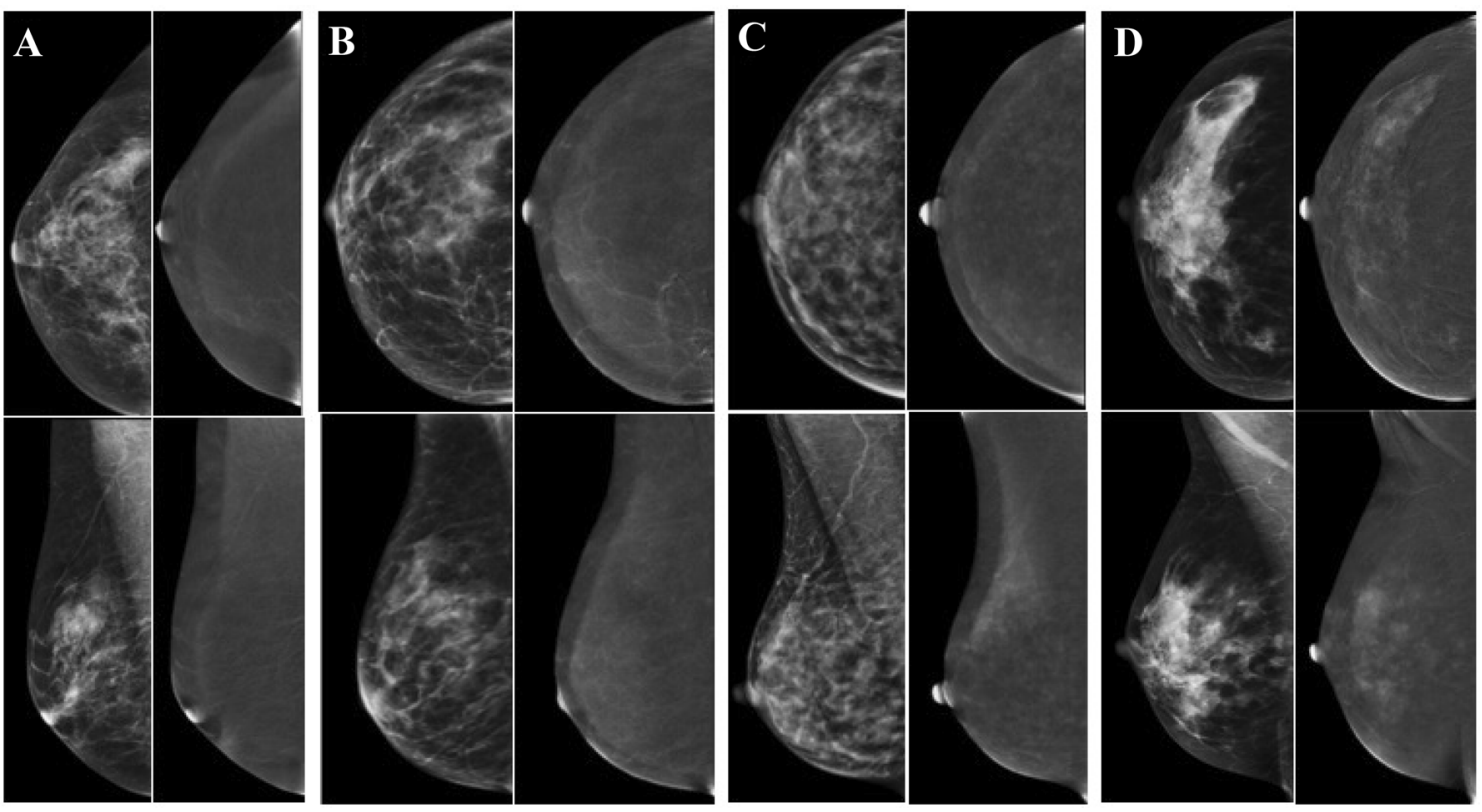

Fig. 2 Background enhancement on CEM. A Minimal BPE, B mild BPE, C moderate BPE, D marked BPE

hyperplasia, infection, and inflammatory lesions [25] may not be easily diagnosed on imaging and require tissue sampling for confirmation. Additionally, some malignancies demonstrate little to no enhancement on MRI and presumably CEM [26].

Currently, there is no standard BI-RADS lexicon for interpreting and reporting CEM images. As of now, the proposed language for CEM includes the use of mammography BI-RADS lexicon for low-energy images and MRI BI-RADS lexicon for enhancement-related findings.

\section{Comparing the Diagnostic Performance of CEM to Ultrasound, Mammogram, and MRI}

Diagnostic workup for an imaging finding or area of clinical concern begins with obtaining different mammographic projections followed by a sonographic assessment. If further characterization is required, an MRI is typically performed. As CEM provides similar diagnostic information, the question is raised whether it can be performed instead of MRI in certain situations. Multiple studies have shown that the low-energy CEM images are non-inferior to full-field digital mammography [22•,27]. A recent study by 
Fig. 3 Presentation of masses on CEM as an area of abnormal enhancement. A Solid enhancing mass, $\mathbf{B}$ peripherally enhancing mass, $\mathbf{C}$ complex cystic and solid mass with nodular enhancement (arrow)
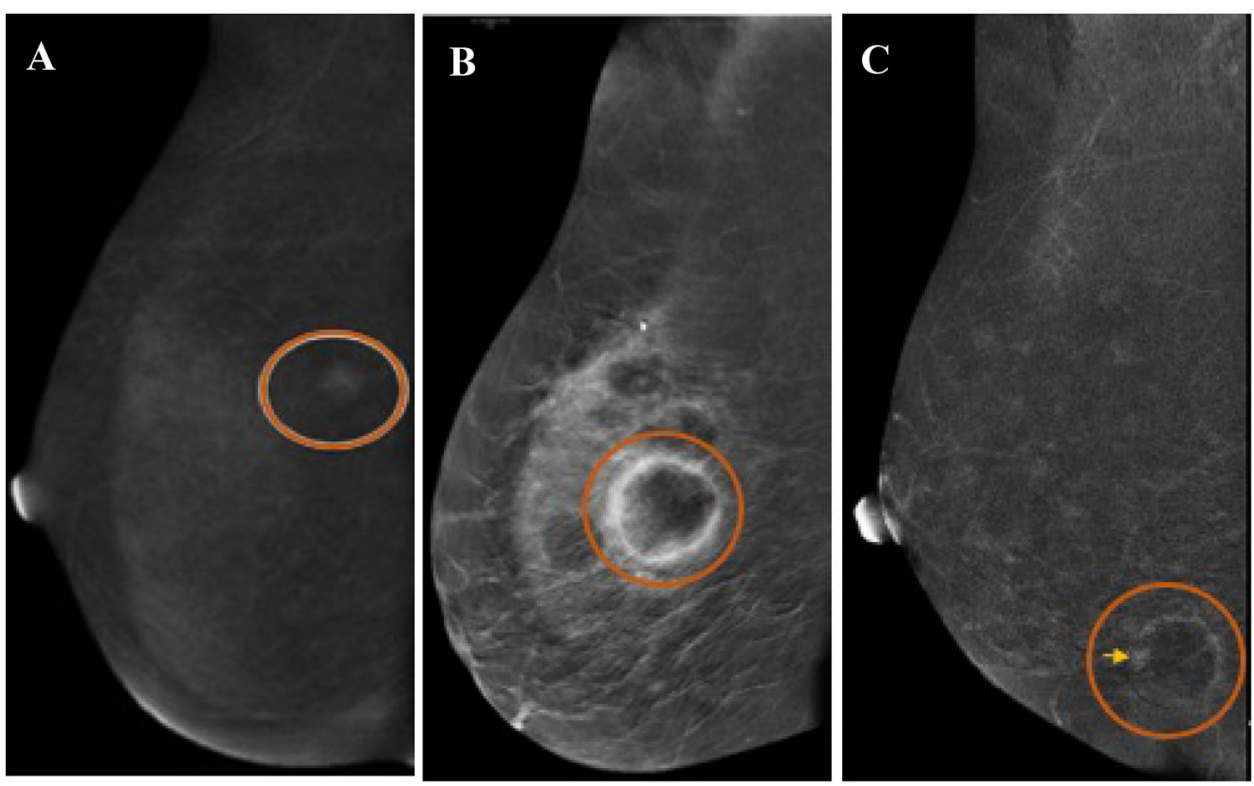

an Egyptian group including 82 patients compared the diagnostic performance of CEM and MRI. The results were comparable with Fallenberg et al. [28], showing clinically significant higher sensitivities and negative predictive values (NPV) with MRI compared to CEM (100\% vs. 94\% in sensitivity $[p=0.014]$ vs. 100 vs. $82.5 \%$ in NPV $[p=0.013]$ ), but no significant difference in specificity, positive predictive value (PPV), and accuracy [29]. Ultrasound is typically still utilized regardless as it provides complementary information, specifically if the target is accessible for ultrasound-guided biopsy. Additional studies are needed to determine which imaging findings or patient populations could be effectively evaluated with CEM. Table 1 summarizes the current literature comparing the diagnostic performance of CEM to digital mammography, sonography, and MRI in various clinical settings. Notably, sensitivity and specificity data between CEM and MRI are comparable across multiple studies. While Table 1 may also suggest that ultrasound is comparable to CEM and MRI, ultrasound as a stand-alone modality is not as commonly studied as the other modalities. More data are likely needed before accepting ultrasound as a stand-alone modality.

\section{Assessment of Masses}

Many lesions, whether benign or malignant, may present on CEM as an enhancing mass. Data from a Dutch group in 2016 studying CEM evaluation of 199 screening recall found 152 out of 199 findings (76\%) were masses [34]. Based on prior studies, they assumed that low-energy images be equivalent to digital mammography. They found increased sensitivity $(93.0 \%$ to $96.9 \%)$ and specificity
(35.9\% to $69.7 \%$ ) when comparing mammography vs. CEM for evaluation of masses. Currently, most masses are evaluated with a diagnostic mammogram followed by targeted ultrasound. To our knowledge, there have been no specific studies comparing the characterization of masses with diagnostic mammography and ultrasound versus CEM, presumably due to the ease of sonographic characterization. While CEM may not play a significant role in characterizing a mass, the ability to evaluate the remainder of the breasts for multifocal, multicentric, or contralateral disease remains useful, particularly in staging.

In most cases, a solid, enhancing mass will be further evaluated on ultrasound and most likely biopsied for pathology confirmation (Fig. 3A). A circumscribed mass with a thin rim of peripheral enhancement without any central-enhancing component probably corresponds to a simple inflamed cyst. This thin rim of enhancement is known as the "eclipse" sign [42] (Fig. 3B). This finding, however, should be confirmed on low-energy images with possible ultrasound to exclude a complex cystic and solid mass. A complex cystic and solid mass may demonstrate thick, asymmetric, or nodular rim enhancement, possibly with non-enhancing central components (Fig. 3C). Ultrasound with a possible biopsy is always warranted in this case.

\section{Assessment of Architectural Distortion}

Architectural distortion (AD) is one of the most overlooked findings on screening mammography, with an estimated prevalence of about $6 \%$ [43]. AD represents various benign and malignant entities, including post-surgical distortion, radial scar, trauma/infection, or breast cancer. In one study 


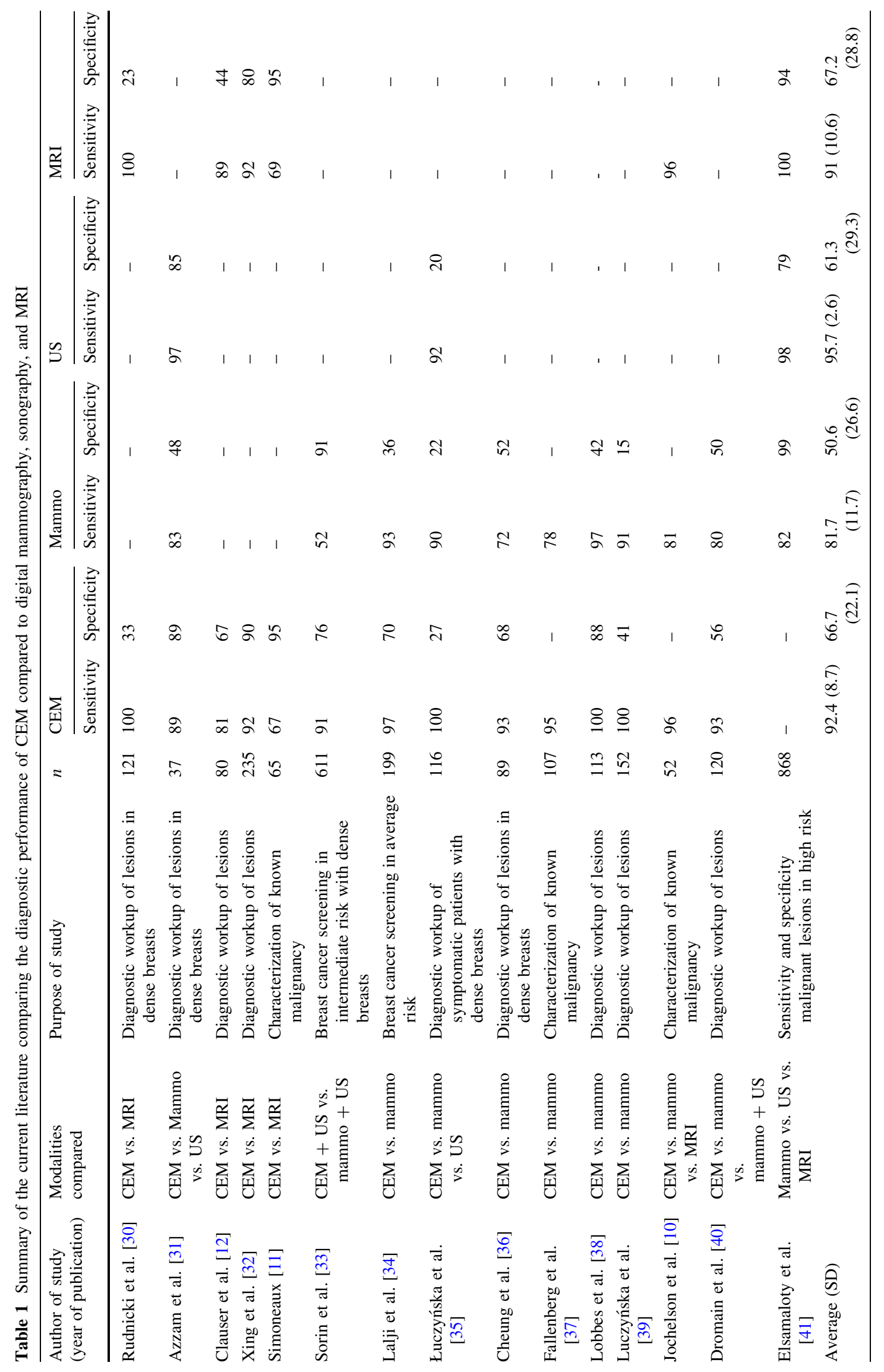


over ten years, including over 200,000 patients, the sole finding of $\mathrm{AD}$ was estimated to have PPV of $74.5 \%$ for malignancy (274/369) [44]. The same study found that in the absence of a sonographic correlate, the likelihood of malignancy is lower but remains greater than $20 \%$. The addition of tomosynthesis has allowed for greater confidence in detecting AD. However, it has been reported that the PPV of AD found on tomosynthesis can be over 50\%, particularly if the $\mathrm{AD}$ is occult on conventional mammography views [45]. MRI has also been a valuable adjunct for further characterization of these lesions. Some have suggested benignity based on the absence of enhancement post-contrast administration and specific threshold ADC values [46]. As CEM also characterizes lesion enhancement, it could likely be used in place of MRI for diagnostic characterization. Patel et al. studied a small group of patients with a total of 49 cases of AD identified on tomosynthesis images to determine if CEM could effectively exclude malignancy [47]. Out of 49 lesions, 30 were identified as malignant, with the remainder representing benign lesions ( 9 radial scar, 10 other benign pathologies), with 37 out of 49 lesions demonstrating enhancement. High sensitivity and NPV were noted at $96.7 \%$ and $91.7 \%$, respectively, suggesting that CEM could be useful in risk stratifying for biopsy in the setting of AD. Given that 1 of the $12(8.3 \%)$ non-enhancing lesions eventually led to a cancer diagnosis, the absence of enhancement does not necessarily exclude the presence of malignancy, and more extensive studies are needed for further evaluation.

\section{Assessment of Microcalcifications}

Microcalcifications are commonly depicted on low-energy images and are characterized based on their imaging appearance and distribution, similar to conventional mammography. CEM allows for additional characterization of the calcifications according to the extent and magnitude of enhancement characteristics on the recombined images, potentially stratifying the calcifications into different risk categories for malignancy. Few studies have evaluated the potential role that CEM has in the characterization of microcalcifications. Cheung et al. [48] analyzed a total of 94 lesions that were diagnosed as suspicious for malignancy (BI-RADS 4) and found 27 malignancies, 32 pre-malignant lesions, and 35 benign lesions, with 33 enhancing lesions (35\%) and 61 without enhancement (65\%). Of the malignancies, all biopsies with invasive ductal carcinoma demonstrated enhancement (8 cases; $100 \%$ of invasive cancers), with 16 of 19 biopsies with DCIS showing enhancement (84\% of DCIS cases). A larger study by Houben et al. [49] found enhancement in $84.4 \%$ cases of invasive ductal carcinoma (27 of 32 ) and
$81.1 \%$ of DCIS (27 of 33). Based on this data, the presence of enhancement suggests malignancy. However, the absence of enhancement in the setting of suspicious calcifications does not exclude malignancy, and biopsy should still be pursued.

\section{Assessment of Non-mass Enhancement (NME)}

Focal or linear enhancement above BPE on CEM is managed similar to NME on MRI. In the absence of a corresponding finding on low-energy CEM images, ultrasound, and MRI, the presence of malignancy is less likely but theoretically possible. To our knowledge, no study has characterized the incidence of focal enhancement on CEM without a correlating lesion on another modality. False positives may be related to focal background enhancement, which is, for whatever reason, more conspicuous compared to the surrounding parenchyma. A study by Chikarmane and colleagues including 205 women showed that up to $20 \%$ of cases of NME on MRI were reclassified as BPE on re-evaluation, with only 1 out of the 77 patients that were reclassified ultimately being diagnosed as a malignancy on pathology [50]. Subtle AD, including radial scars or invasive lobular carcinoma, tend to be more elusive on mammography and typically better characterized on MRI. Prior research has shown that $\mathrm{AD}$ depicted on screening mammography without an imaging correlate on diagnostic mammography or ultrasonography is less likely to be malignant [51, 52]. Additional research is needed to determine if these enhancing lesions on CEM without a discrete correlating lesion on other modalities are associated with underlying early or developing pathology. Figure 4 and Fig. 5 demonstrate two cases of abnormal NME on CEM with subsequent biopsy yielding invasive cancer.

\section{Applications}

\section{Screening}

While mammography has been the accepted standard for breast cancer screening in women with an average risk for developing breast cancer, studies have shown decreased sensitivity in women with dense breasts [53, 54]. In addition, populations with an intermediate to high $(>20 \%)$ lifetime risk of breast cancer can benefit from supplemental screening. MRI has been used to fulfill this role for the past 30 years due to improved sensitivity. However, it has been associated with increased false-positive rates, unnecessary biopsies, and increased costs [55, 56]. Multiple studies have shown that CEM outperforms standard digital mammography in the detection of malignancy $[36,37,57]$ with sensitivities comparable to that of MRI (CEM: 94-100\% 

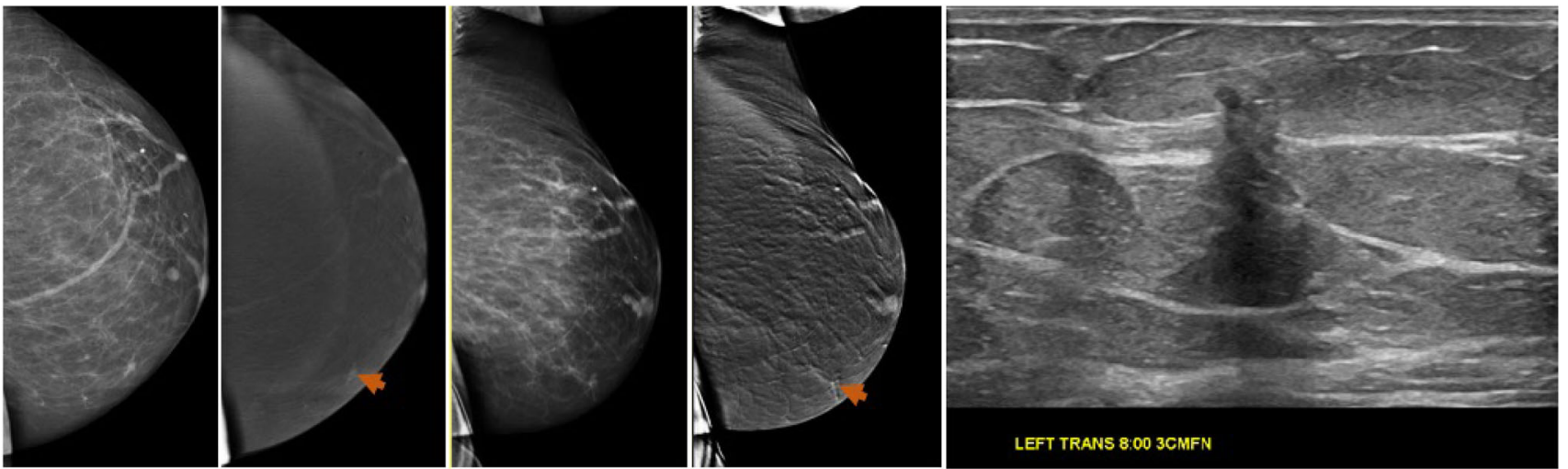

Fig. 462 years old, history of right breast cancer, status postconservation therapy for screening. Focal area of nonmass enhancement 8:00 axis left breast without correlate on conventional mammographic views. Sonographic evaluation of the area showed an irregular mass. Ultrasound-guided core needle biopsy yielded invasive ductal carcinoma
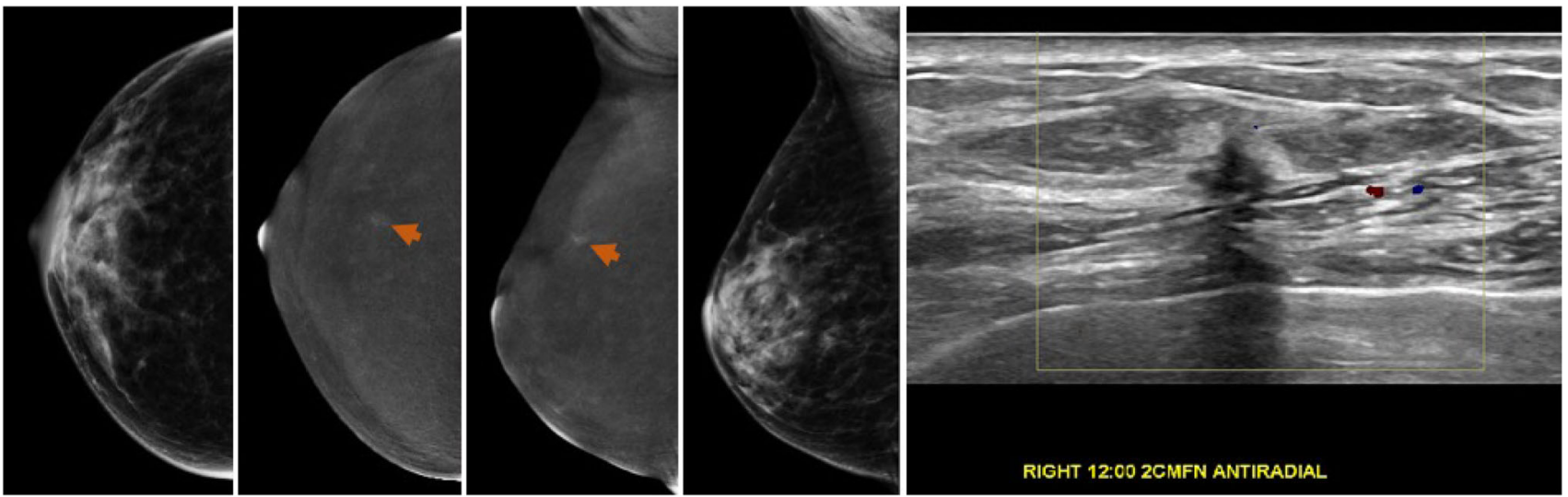

Fig. 556 years old, history of left breast cancer, status post-left mastectomy for screening. Focal area of non-mass enhancement 12 o'clock right breast without correlate on conventional mammographic

vs. MRI: 93-99\%) [58-60]. In an initial study by Jochelson et al. [61], they discovered a total of 3 cancers in a cohort of 307 patients with an intermediate- to high-risk category. Digital mammography did not detect any malignancy, CEM detected 2 out of 3 cancers (both invasive cancer), and MRI detected all 3 (two invasive CA and 1 DCIS). CEM demonstrated a PPV of $15.4 \%$ (95\% CI [1.9-45.4], 2 out of 13 biopsies) with specificity of $94.7 \%$ (95\% CI [91.6-97]), compared to PPV of $14.3 \%$ (95\% CI [3.0-36.3\%] 3 out of 11 biopsies) and specificity of $94.1 \%$ (95\% CI [90.8-96.4]) for MRI. A larger follow-up study including nearly 1200 patients with a similar intermediate to high risk for breast cancer showed CEM to have a PPV of $20.9 \%$ (95\% CI [11.9, 32.6], 14 out of 67 biopsies) [62・•], ultimately concluding that CEM could potentially be performed for both primary and supplemental screening.

In addition to similar sensitivities with decreased falsepositive rates compared to MRI, CEM has additional advantages in screening. Background enhancement is typically less problematic when interpreting CEM compared views. Sonographic evaluation of the area showed an irregular mass. Ultrasound-guided core needle biopsy yielded invasive lobular carcinoma

to MRI. Li et al. [63] compared the enhancement intensity of background parenchyma between CEM and MRI and showed that BPE is significantly lower for CEM when compared to MRI $(P<0.01)$. They also showed that the intensity of lesion enhancement was considerably lower on CEM than MRI $(P<0.01)$. Lower breast background enhancement might contribute to a higher PPV and a lower false-positive rate compared to MRI. CEM has the potential for significant cost savings for the patient as well as the health system. Data suggest that if CEM was used instead of MRI for high-risk supplemental screening instead of MRI, the estimated cost savings could approach 1.1 billion dollars annually [64]. Other reports have found that some women would prefer to undergo CEM rather than MRI due to a combination of factors, including relatively shorter acquisition times and improved patient comfort $[65,66]$. In some instances, when MRI is contraindicated due to MRIincompatible devices/implants, weight limitations, or significant imaging artifacts, CEM could be considered a firstline alternative without sacrificing sensitivity. While using 
ionizing radiation is not ideal in a screening population that is intrinsically at high risk for malignancy, the prolonged use of gadolinium deposition has come into question in recent years [67], which needs to be considered given that screening could potentially span decades.

Given the comparable diagnostic performance of CEM versus MRI, some groups have evaluated the potential role of CEM in screening populations with intermediate breast cancer risk, defined by the ACR as those with a 15-20\% lifetime risk of developing breast cancer. Intermediate risk typically includes women with a personal or family history of breast cancer, those with previous biopsy demonstrating atypia or lobular carcinoma in situ (LCIS), and those with dense breasts [68]. Sorin et al. [33] studied a group of 611 consecutive intermediate risk with nearly $50 \%$ of patients with a personal or family history of breast cancer and over $90 \%$ with dense breasts, diagnosing a total of 21 cancers. CEM demonstrated superior sensitivity and specificity compared to digital mammography $(90.5 \%$ vs. $52.4 \%$ and $90.5 \%$ vs. $76.1 \%$, respectively) with an incremental cancer detection rate of 13.1 per 100 women (95\% CI [6.1-20.1]). Interestingly, an adjunct whole-breast ultrasound was performed for negative screeners by CEM, leading to 73 additional suspicious findings, all of which were ultimately determined to be false positives. They concluded that CEM was significantly more sensitive than digital mammography in this intermediate population. There was no added benefit for performing screening whole-breast ultrasound in those deemed negative by CEM screening. A recent study of 132 women with a personal history of lobular neoplasia (atypical lobular hyperplasia or LCIS) detected a total of 6 malignancies with sensitivity and NPV of $100 \%$ with specificity and accuracy of $88 \%$. It was concluded that although a larger population should be studied to ascertain the true diagnostic performance, CEM screening in this subset of patients is very promising [69].

\section{Diagnostic Evaluation}

Multiple studies have demonstrated the added value that CEM provides for the evaluation of screening abnormalities. A European study evaluated the diagnostic performance of CEM in 199 consecutive average-risk patients who were called back from screening mammogram. They demonstrated superior diagnostic performance of CEM compared to convention mammography in this setting. Even when less experienced readers use CEM, there is an increase in all diagnostic accuracy parameters, especially specificity and positive predictive value for breast cancer [34].

In a follow-up study including 116 women recommended for a callback from screening, the largest improvements were detected while assessing specificity and PPV, which increased from $42.0 \%$ and $39.7 \%$ to $87.7 \%$ and $76.2 \%$, respectively [38]. In the patients with a final diagnosis of malignancy, the authors found that tumor size was consistent between CEM, MRI, and pathology. Based on the significant improvements in specificity and PPV, it was concluded that CEM could improve the false-positive rate relative to mammography alone.

A recent study performed a systematic review of 8 prospective trials assessing CEM, including in the metaanalysis a total of 945 lesions [70]. They reported a summary area under the curve obtained from all the studies of $89 \%$ [95\% CI $86 \%-91 \%$ ], with a sensitivity of $85 \%$ [95\% CI $73 \%-93 \%$ ] and a specificity of $77 \%$ [95\% CI $60 \%-$ $88 \%$ ]. This study concluded that CEM could be considered only for diagnostic evaluation of indeterminate breast findings when sonographic and conventional mammographic studies are inconclusive, or MRI is contraindicated or not available.

Xing et al. compared the diagnostic performance of CEM with MRI in 235 patients with suspected breast abnormalities based on clinical examination or mammography [32]. They demonstrated a better accuracy and specificity for CEM in breast cancer detection when compared to MRI. Interestingly, the false-positive rate was lower for CEM (10.5\%) compared to MRI (19.8\%). CEM maintained comparable sensitivity, positive predictive value, negative predictive value, and false-negative rate (91.5\%, 94.7\%, 83.7\%, and $8.5 \%$ vs. $91.5 \%, 90.5 \%$, $82.1 \%$, and $8.5 \%$ ).

In summary, current data support the application of CEM as a valuable problem-solving imaging modality in the setting of recalls from the breast cancer screening program and in the diagnostic setting.

\section{Evaluating the Extent of Disease}

Staging breast cancer is a critical component for the appropriate treatment planning to assess the extent of disease in the ipsilateral breast or synchronous cancer in the contralateral breast. The modalities used for staging vary between institutions, and what imaging protocols constitute adequate staging remains controversial. Comparisons between CEM, digital mammography, US, and MRI in characterizing lesion extent have been studied by multiple authors. Łuczyńska et al. [35] characterized 225 known lesions by CEM and digital mammography relative to histologic samples. They found that CEM was significantly more sensitive than digital mammography alone to detect malignancy $(100 \%$ vs. $90 \%)$, with enhancement in the presence of a mass or calcifications being highly suggestive of malignancy. Compared to histology, lesions were only slightly overestimated in size by $2-3 \mathrm{~mm}$, and it was concluded that CEM could provide accurate lesion 
characterization within a few millimeters. In 2017, Patel et al. [71] also compared the characterization of tumor extent by CEM, US, and digital mammography relative to histology in a cohort of 88 patients with a recent breast cancer diagnosis. Overall, CEM demonstrated the strongest correlation to histology followed by US and digital mammography after analyzing Pearson correlation coefficients (0.859, 0.649, 0.598, respectively, $P<0.001)$. When further stratifying patients based on breast density into dense and non-dense breasts, it was noted that histopathologic size correlated more closely with CEM compared to digital mammography (Pearson correlation coefficient of 0.843 vs. 0.555 in dense breasts compared to 0.885 vs. 0.618 in nondense breasts). CEM was noted to slightly overestimate lesion size by $3 \mathrm{~mm}$, while US and digital mammography underestimated tumor size by 1-3 $\mathrm{mm}$. It was subsequently concluded that CEM could be an effective tool for preoperative tumor measurement, particularly in dense breasts or in those for whom MRI is contraindicated. With regard to MRI, multiple studies have shown that CEM has similar sensitivity and diagnostic accuracy with comparable estimates of tumor size [10••, 59, 60], again noting that CEM slightly overestimates tumor size compared to final pathology. Fallenberg et al. [59] showed that CEM more closely estimates tumor size in 80 patients with recently diagnosed breast cancer than histologic references. However, no significant difference in absolute measurements was seen between CEM and MRI, suggesting that they could be used interchangeably. MRI continues to be more sensitive to evaluating the chest wall and axilla, which is not well assessed on CEM since it is a projection modality.

\section{Monitoring Treatment Response}

With neoadjuvant chemotherapy being used more frequently, accurate imaging to assess for clinical response and extent of residual disease is crucial in optimizing patient outcomes. While a combination of mammograms and ultrasound and MRI have been used to evaluate changes related to treatment, there is increasing interest to determine if CEM can also play a role in characterizing residual disease. A study involving a cohort of 21 women diagnosed with Stage II to III breast cancer found that while CEM had $100 \%$ sensitivity at finding complete pathologic response, a total of 6 out of 9 false negatives were seen with minimal residual enhancement on imaging and residual disease on histopathology [72]. Others have reported that while both CEM and MRI underestimated the extent of residual disease, CEM has better sensitivity for detecting complete pathologic response than MRI [73]. In a group of 65 patients with pathology-proven invasive breast cancer, comparable sensitivities and PPV were observed in detecting residual malignancy [74]. It should be noted that based on current data, MRI is not nearly specific enough to completely exclude residual disease after neoadjuvant treatment [75] and the same likely applies to CEM. Given the vast amount of additional information provided by MRI, such as diffusion weighting imaging and changes in kinetics, CEM cannot replace MRI for this indication and should be considered an alternative if MRI is not available or contraindicated.

\section{Pitfalls and Challenges}

Despite its utility as a diagnostic tool, some challenges and potential pitfalls need to be considered before practice implementation.

Unlike digital mammography and ultrasonography, CEM requires the use of iodinated contrast agents. Main contraindications to performing the CEM examination are related to the use of iodinated contrast or non-ionizing radiation, including a history of a severe allergy including anaphylactic or anaphylactoid reaction to iodinated contrast, impaired renal function, and pregnancy. Other suggested contraindications include known BRCA mutations due to radiosensitive and MRI being superior for screening in this population and known hyperthyroidism.

Although current literature about contrast-induced nephropathy has been calling into question the true incidence of renal injury from iodinated contrast [76], history of renal disease including renal insufficiency, prior renal transplant, and solitary kidney still need to be considered. Previous history of allergic-like and physiologic reactions to iodinated contrast may also limit usage in some patients. The latest ACR Manual on Contrast Media estimates the incidence of serious anaphylactic reactions due to iodinated contrast material to be $0.04 \%$ [14]. If CEM is used as a supplemental imaging modality, the risks of premedication may not outweigh the benefits compared to other modalities. Facilities performing CEM would also need to ensure adequate staff training in identifying and managing contrast reactions, stock and maintain necessary equipment and therapeutic interventions, and require an on-site physician to address complications as they arise.

Unlike MRI, CEM lacks the anatomic resolution to provide adequate soft tissue assessment. MRI allows for simultaneous characterization of the bilateral breasts, axilla, and chest wall, critical for the complete staging of malignancy [77]. Additionally, CEM requires the use of non-ionizing radiation. Fallenberg et al. [37] have estimated the average glandular dose per projection at $2.80 \mathrm{mGy}$ compared to $1.78 \mathrm{mGy}$ for standard digital mammography. The increase in dose is related to the fact that two images (low and high $\mathrm{keV}$ ) are required for CEM relative to the 1 per projection in digital mammography. 


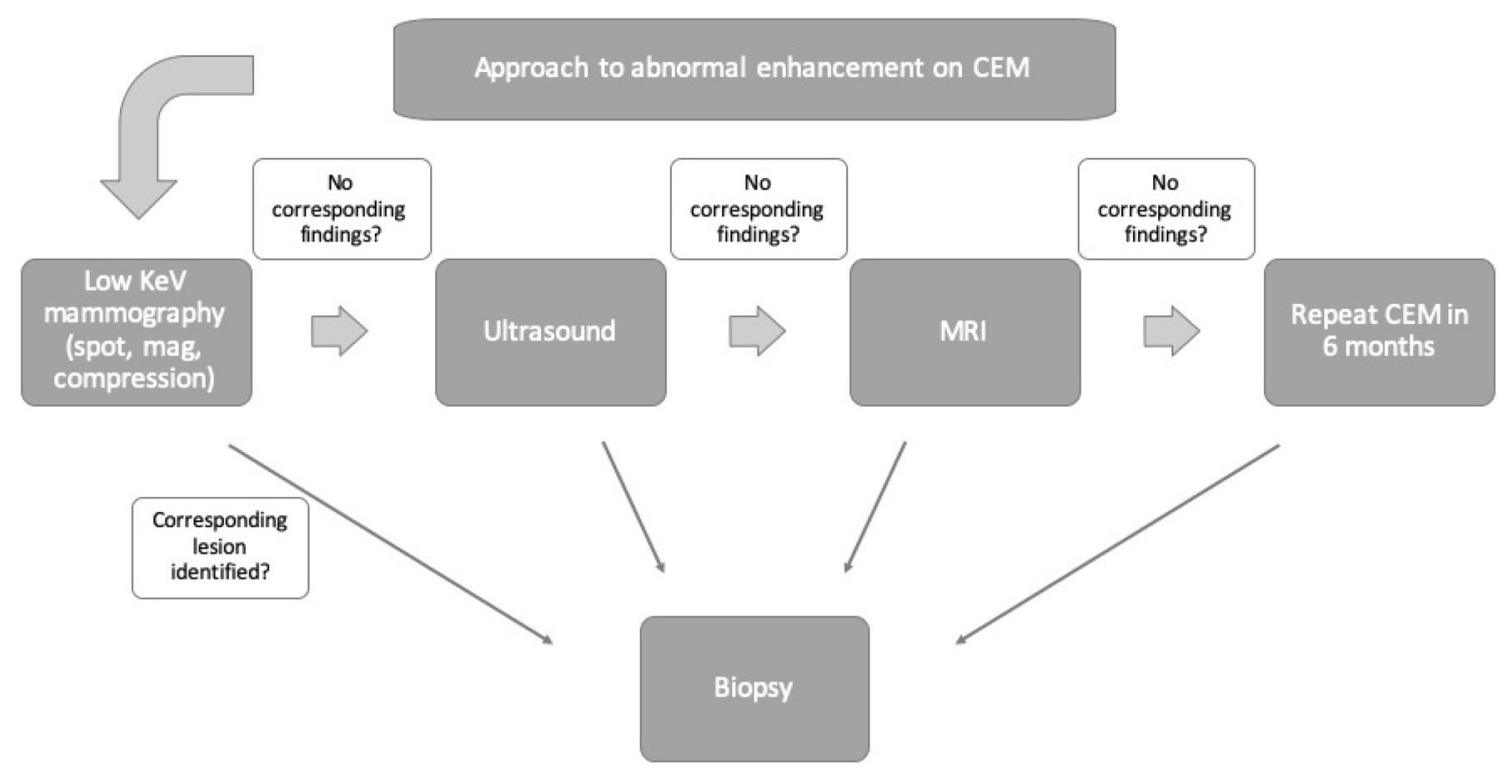

Fig. 6 Workup of an area of abnormal enhancement seen on CEM

Considering data from Hendrick et al. which showed that screening mammography with mean glandular doses ranging from 3.7 to $4.7 \mathrm{mGy}$ contributes to a lifetime attributable risk of radiation-induced fatal breast cancer of approximately $20-25$ cases per 100,000 women screened between the ages of 40 and 80 [78], the benefits likely outweigh the risks.

The American College of Radiology Breast ImagingReporting and Data System (5th edition) does not include terminology for CEM, making it difficult to interpret and compare it to other imaging modalities. Current interpretation relies on a combination of terms already in use in the BI-RADS lexicon for mammography and MRI. Future research is required to refine the terminology further and determine how reliable these terms are across modalities.

CEM-guided biopsy is not readily accessible. Some groups have reported a two-procedure approach with initial mammographic localization of a marker followed by stereotactic biopsy [79], although this may not be clinically feasible in many practice settings. Most commonly, any suspicious imaging findings on the recombined images are further characterized on other modalities, including digital mammography, ultrasound, or MRI (Fig. 6). It is essential to consider that some suspicious lesions noted on CEM may lead to additional workup for patients with ultrasound or MRI, which previously would have been "normal" by digital mammography, potentially increasing costs and patient anxiety. As of June 2020, GE healthcare has received FDA clearance for the 1st CEM-guided biopsy system (Serena Bright). Early reports suggest that some patients prefer CEM-guided over MR-guided biopsies. However, larger studies still need to be conducted. Another biopsy option currently being explored is using an upright stereotactic unit in a CEM-capable mammogram unit and performing the biopsy immediately following the acquisition of CEM images. In this setting, the radiologist can target the area of suspicious enhancement on the upright stereotactic unit. If CEM-guided biopsy becomes clinically accessible, the costs of cross-modality workup could be potentially subverted.

Similar to MRI, CEM cannot readily distinguish benign from malignant lesions, with false and negative results being relatively common. Multiple benign histology, including fibroadenoma, papilloma, PASH, and abscess, is typically associated with enhancement and may be challenging to differentiate via imaging [80]. Smaller studies have shown that up to $16 \%$ of DCIS will not demonstrate enhancement on CEM [48].

\section{Conclusion}

CEM is an emerging problem-solving breast imaging modality that provides complementary information to conventional imaging modalities and may potentially be used in place of MRI for specific indications and/or patient populations.

\section{Declarations}

Conflict of interest Drs. Hannsun, Elmi, Sylvan, and Saponaro declare no potential conflicts of interest.

Research Involving Human and Animal Rights This article does not contain any studies with human or animal subjects performed by any of the authors. 
Open Access This article is licensed under a Creative Commons Attribution 4.0 International License, which permits use, sharing, adaptation, distribution and reproduction in any medium or format, as long as you give appropriate credit to the original author(s) and the source, provide a link to the Creative Commons licence, and indicate if changes were made. The images or other third party material in this article are included in the article's Creative Commons licence, unless indicated otherwise in a credit line to the material. If material is not included in the article's Creative Commons licence and your intended use is not permitted by statutory regulation or exceeds the permitted use, you will need to obtain permission directly from the copyright holder. To view a copy of this licence, visit http://creativecommons. org/licenses/by/4.0/.

\section{References}

Papers of particular interest, published recently have been highlighted as:

- Of importance

-• Of major importance

1. Fletcher SW, Elmore JG. Clinical practice. Mammographic screening for breast cancer. N Engl J Med. 2003;348:1672-80. https://doi.org/10.1056/NEJMcp021804.

2. Pisano ED, Gatsonis C, Hendrick E, et al. Diagnostic performance of digital versus film mammography for breast-cancer screening. N Engl J Med. 2005;353:1773-83. https://doi.org/10. 1056/nejmoa052911.

3. Humphrey LL, Helfand M, Chan BKS, Woolf SH. Breast cancer screening: a summary of the evidence for the U.S. preventive services task force. Ann Intern Med. 2002;137:347-60. https:// doi.org/10.7326/0003-4819-137-5_part_1-200209030-00012.

4. Kolb TM, Lichy J, Newhouse JH. Comparison of the performance of screening mammography, physical examination, and breast US and evaluation of factors that influence them: an analysis of 27,825 patient evaluations. Radiology. 2002;225:165-75. https://doi.org/10.1148/radiol.2251011667.

5. Zhang Y, Ren H. Meta-analysis of diagnostic accuracy of magnetic resonance imaging and mammography for breast cancer. J Cancer Res Ther. 2017;13:862-8. https://doi.org/10.4103/jcrt. JCRT_678_17.

6. Day D. Breast MRI: opportunities and challenges. Minn Med. 2009;92:45-8.

7. Watt AC, Ackerman LV, Shetty PC, et al. Differentiation between benign and malignant disease of the breast using digital subtraction angiography of the breast. Cancer. 1985;56:1287-92. https://doi.org/10.1002/1097-0142(19850915)56:6\%3c1287: AID-CNCR2820560611\%3e3.0.CO;2-L.

8. Skarpathiotakis M, Yaffe MJ, Bloomquist AK, et al. Development of contrast digital mammography. Med Phys. 2002;29:2419-26. https://doi.org/10.1118/1.1510128.

9. Lewin JM, Isaacs PK, Vance V, Larke FJ. Dual-energy contrastenhanced digital subtraction mammography: feasibility. Radiology. 2003;229:261-8. https://doi.org/10.1148/radiol.2291021276.

10. • Jochelson MS, Dershaw DD, Sung JS, et al. Bilateral contrastenhanced dual-energy digital mammography: feasibility and comparison with conventional digital mammography and MR imaging in women with known breast carcinoma. Radiology. 2013;266:743-51. https://doi.org/10.1148/radiol.12121084. This study is one of the landmarks studies demonstrating comparable performance for CEM for detecting breast cancer at to that of MRI and higher than that of conventional digital mammography.
11. Simoneaux R. Comparing contrast-enhanced spectral mammography \& MRI. Oncol Times. 2018;40:6-7. https://doi.org/10. 1097/01.cot.0000530516.08587.86.

12. Clauser P, Baltzer PAT, Kapetas P, et al. Low-dose, contrastenhanced mammography compared to contrast-enhanced breast MRI: a feasibility study. J Magn Reson Imaging. 2020;52:589-95. https://doi.org/10.1002/jmri.27079.

13. Ducote JL, Molloi S. Quantification of breast density with dual energy mammography: an experimental feasibility study. Med Phys. 2010;37:793-801. https://doi.org/10.1118/1.3284975.

14. ACR Committee on drugs and contrast Media. ACR Manual on Contrast Media. ACR Man Contrast Media - Version 9, 2013 105:128. https://www.acr.org/-/media/ACR/Files/ClinicalResources/Contrast_Media.pdf (2013)

15. Shaw DD, Potts DG. Toxicology of iohexol. Investig Radiol. 1985;20:S10-3. https://doi.org/10.1097/00004424-19850100200004.

16. Ralston WH, Robbins MS, James P. Reproductive, developmental, and genetic toxicology of ioversol. Investig Radiol. 1989;24:S16-23. https://doi.org/10.1097/00004424-19890600100004.

17. James JJ, Tennant SL. Contrast-enhanced spectral mammography (CESM). Clin Radiol. 2018;73:715-23. https://doi.org/10.1016/j. crad.2018.05.005.

18. Lewis TC, Pizzitola VJ, Giurescu ME, et al. Contrast-enhanced digital mammography: a single-institution experience of the first 208 cases. Breast J. 2017;23:67-76. https://doi.org/10.1111/tbj. 12681.

19. Koukou V, Martini N, Fountos G et al. Calcification detection optimization in dual energy mammography: influence of the X-ray spectra. In: IFMBE proceedings. Springer; 2014. pp. 459-462. https://doi.org/10.1007/978-3-319-00846-2_114

20. Jeukens CRLPN. Physics of contrast-enhanced mammography. In: Contrast-enhanced mammography. Springer; 2019. pp. 23-39. https://doi.org/10.1007/978-3-030-11063-5_2

21. Zanardo M, Cozzi A, Trimboli RM, et al. Technique, protocols and adverse reactions for contrast-enhanced spectral mammography (CESM): a systematic review. Insights Imaging. 2019. https://doi.org/10.1186/s13244-019-0756-0.

22. - Francescone MA, Jochelson MS, Dershaw DD, et al. Low energy mammogram obtained in contrast-enhanced digital mammography (CEDM) is comparable to routine full-field digital mammography (FFDM). Eur J Radiol. 2014;83:1350-5. https:// doi.org/10.1016/j.ejrad.2014.05.015. One of the first studies confirming that low energy CEM images are equivalent to standard digital mammography despite the presence of intravenous iodinated contrast. Low energy CEDM images may be used for interpretation in place of the mammogram, thereby reducing patient dose.

23. Sogani J, Morris EA, Kaplan JB, et al. Comparison of background parenchymal enhancement at contrast-enhanced spectral mammography and breast MR imaging. Radiology. 2017;282:63-73. https://doi.org/10.1148/radiol.2016160284.

24. Savaridas SL, Taylor DB, Gunawardana D, Phillips M. Could parenchymal enhancement on contrast-enhanced spectral mammography (CESM) represent a new breast cancer risk factor? Correlation with known radiology risk factors. Clin Radiol. 2017;72:1085.e1-1085.e9. https://doi.org/10.1016/j.crad.2017.07. 017.

25. Mario J, Venkataraman S, Dialani V, Slanetz PJ. Benign breast lesions that mimic cancer: determining radiologic-pathologic concordance. Appl Radiol. 2015;44:24-32. https://doi.org/10. 1186/s13244-019-0756-0.

26. Ghai S, Muradali D, Bukhanov K, Kulkarni S. Nonenhancing breast malignancies on MRI: sonographic and pathologic 
correlation. Am J Roentgenol. 2005;185:481-7. https://doi.org/ 10.2214/ajr.185.2.01850481.

27. Lalji UC, Jeukens CRLPN, Houben I, et al. Evaluation of lowenergy contrast-enhanced spectral mammography images by comparing them to full-field digital mammography using EUREF image quality criteria. Eur Radiol. 2015;25:2813-20. https://doi. org/10.1007/s00330-015-3695-2.

28. Fallenberg EM, Schmitzberger FF, Amer H, et al. Contrast-enhanced spectral mammography vs. mammography and MRIclinical performance in a multi-reader evaluation. Eur Radiol. 2017;27:2752-64. https://doi.org/10.1007/s00330-016-4650-6.

29. Kamal RM, Hanafy MM, Mansour SM, et al. Can contrast-enhanced mammography replace dynamic contrast-enhanced MRI in the assessment of sonomammographic indeterminate breast lesions? Egypt J Radiol Nucl Med. 2020. https://doi.org/10.1186/ s43055-020-00188-0.

30. Rudnicki W, Piegza T, Rozum-Liszewska N, et al. The effectiveness of contrast-enhanced spectral mammography and magnetic resonance imaging in dense breasts. Pol $\mathrm{J}$ Radiol. 2021;86:e159-64. https://doi.org/10.5114/pjr.2021.104834.

31. Azzam H, Kamal RM, Hanafy MM, et al. Comparative study between contrast-enhanced mammography, tomosynthesis, and breast ultrasound as complementary techniques to mammography in dense breast parenchyma. Egypt J Radiol Nucl Med. 2020;51:148. https://doi.org/10.3348/kjr.2014.15.6.689.

32. Xing D, Lv Y, Sun B, et al. Diagnostic value of contrast-enhanced spectral mammography in comparison to magnetic resonance imaging in breast lesions. J Comput Assist Tomogr. 2019;43:245-51. https://doi.org/10.1097/RCT.000000000000 0832.

33. Sorin V, Yagil Y, Yosepovich A, et al. Contrast-enhanced spectral mammography in women with intermediate breast cancer risk and dense breasts. Am J Roentgenol. 2018. https://doi. org/10.2214/AJR.17.19355.

34. Lalji UC, Houben IPL, Prevos R, et al. Contrast-enhanced spectral mammography in recalls from the Dutch breast cancer screening program: validation of results in a large multireader, multicase study. Eur Radiol. 2016;26:4371-9. https://doi.org/10. 1007/s00330-016-4336-0.

35. Łuczyńska E, Niemiec J, Hendrick E, et al. Degree of enhancement on contrast enhanced spectral mammography (CESM) and lesion type on mammography (MG): comparison based on histological results. Med Sci Monit. 2016;22:3886-93. https://doi. org/10.12659/MSM.900371.

36. Cheung YC, Lin YC, Wan YL, et al. Diagnostic performance of dual-energy contrast-enhanced subtracted mammography in dense breasts compared to mammography alone: interobserver blind-reading analysis. Eur Radiol. 2014;24:2394-403. https:// doi.org/10.1007/s00330-014-3271-1.

37. Fallenberg EM, Dromain C, Diekmann F, et al. Contrast-enhanced spectral mammography: does mammography provide additional clinical benefits or can some radiation exposure be avoided? Breast Cancer Res Treat. 2014;146:371-81. https://doi. org/10.1007/s10549-014-3023-6.

38. Lobbes MBI, Lalji U, Houwers $\mathrm{J}$, et al. Contrast-enhanced spectral mammography in patients referred from the breast cancer screening programme. Eur Radiol. 2014;24:1668-76. https://doi. org/10.1007/s00330-014-3154-5.

39. Luczyńska E, Heinze-Paluchowska S, Dyczek S, et al. Contrastenhanced spectral mammography: comparison with conventional mammography and histopathology in 152 women. Korean $\mathrm{J}$ Radiol 2014;15(6):689-96. https://doi.org/10.3348/kjr.2014.15.6. 689.

40. Dromain C, Thibault F, Muller S, et al. Dual-energy contrastenhanced digital mammography: initial clinical results. Eur
Radiol. 2011;21:565-74. https://doi.org/10.1007/s00330-0101944-y.

41. Elsamaloty H, Elzawawi MS, Mohammad S, et al. Increasing accuracy of detection of breast cancer with 3-T MRI. Am J Roentgenol. 2009;192:1142-48. https://doi.org/10.2214/AJR.08. 1226.

42. Tennant SL, James JJ, Cornford EJ, et al. Contrast-enhanced spectral mammography improves diagnostic accuracy in the symptomatic setting. Clin Radiol. 2016;71:1148-55. https://doi. org/10.1016/j.crad.2016.05.009.

43. Digabel-Chabay C, Allioux C, Labbe-Devilliers C, et al. Architectural distortion and diagnostic difficulties. J Radiol. 2004;85:2099-106. https://doi.org/10.1016/s0221-0363(04)97 788-8.

44. Bahl M, Baker JA, Kinsey EN, Ghate SV. Architectural distortion on mammography: correlation with pathologic outcomes and predictors of malignancy. Am J Roentgenol. 2015;205:1339-45. https://doi.org/10.2214/AJR.15.14628.

45. Ray KM, Turner E, Sickles EA, Joe BN. Suspicious findings at digital breast tomosynthesis occult to conventional digital mammography: imaging features and pathology findings. Breast J. 2015;21:538-42. https://doi.org/10.1111/tbj.12446.

46. Si L, Zhai R, Liu X, et al. MRI in the differential diagnosis of primary architectural distortion detected by mammography. Diagnos Interv Radiol. 2016;22:141-50. https://doi.org/10.5152/ dir.2016.15017.

47. Patel BK, Naylor ME, Kosiorek HE, et al. Clinical utility of contrast-enhanced spectral mammography as an adjunct for tomosynthesis-detected architectural distortion. Clin Imaging. 2017;46:44-52. https://doi.org/10.1016/j.clinimag.2017.07.003.

48. Cheung YC, Juan YH, Lin YC, et al. Dual-energy contrast-enhanced spectral mammography: enhancement analysis on BIRADS 4 non-mass microcalcifications in ScreenedWomen. PLoS ONE. 2016;11:1-12. https://doi.org/10.1371/journal.pone. 0162740 .

49. Houben IPL, Vanwetswinkel S, Kalia V, et al. Contrast-enhanced spectral mammography in the evaluation of breast suspicious calcifications: diagnostic accuracy and impact on surgical management. Acta Radiol. 2019;60:1110-7. https://doi.org/10.1177/ 0284185118822639 .

50. Chikarmane SA, Michaels AY, Giess CS. Revisiting nonmass enhancement in breast MRI: analysis of outcomes and follow-up using the updated BI-RADS atlas. Am J Roentgenol. 2017;209:1178-84. https://doi.org/10.2214/AJR.17.18086.

51. Knutzen AM, Gisvold JJ. Likelihood of malignant disease for various categories of mammographically detected. Nonpalpable Breast Lesions Mayo Clin Proc. 1993;68:454-60. https://doi.org/ 10.1016/S0025-6196(12)60194-3.

52. Shaheen R, Schimmelpenninck CA, Stoddart L, et al. Spectrum of diseases presenting as architectural distortion on mammography: multimodality radiologic imaging with pathologic correlation. Semin Ultrasound CT MRI. 2011;32:351-62. https://doi.org/ 10.1053/j.sult.2011.03.008.

53. Patel MR, Whitman GJ. Negative mammograms in symptomatic patients with breast cancer. Acad Radiol. 1998;5:26-33. https:// doi.org/10.1016/S1076-6332(98)80008-1.

54. Jackson VP, Hendrick RE, Feig SA, Kopans DB. Imaging of the radiographically dense breast. Radiology. 1993;188:297-301. https://doi.org/10.1148/radiology.188.2.8327668.

55. Kriege M, Brekelmans CTM, Boetes C, et al. Efficacy of MRI and mammography for breast-cancer screening in women with a familial or genetic predisposition. $\mathrm{N}$ Engl $\mathrm{J}$ Med. 2004;351:427-37. https://doi.org/10.1056/nejmoa031759.

56. Berg WA, Zhang Z, Lehrer D, et al. Detection of breast cancer with addition of annual screening ultrasound or a single screening MRI to mammography in women with elevated breast cancer 
risk. JAMA J Am Med Assoc. 2012;307:1394-404. https://doi. org/10.1001/jama.2012.388.

57. Mori M, Akashi-Tanaka S, Suzuki S, et al. Diagnostic accuracy of contrast-enhanced spectral mammography in comparison to conventional full-field digital mammography in a population of women with dense breasts. Breast Cancer. 2017;24:104-10. https://doi.org/10.1007/s12282-016-0681-8.

58. Łuczyńska E, Heinze-Paluchowska S, Hendrick E, et al. Comparison between breast MRI and contrast-enhanced spectral mammography. Med Sci Monit. 2015;21:1358-67. https://doi. org/10.12659/MSM.893018.

59. Fallenberg EM, Dromain C, Diekmann F, et al. Contrast-enhanced spectral mammography versus MRI: initial results in the detection of breast cancer and assessment of tumour size. Eur Radiol. 2014;24:256-64. https://doi.org/10.1007/s00330-0133007-7.

60. Lee-Felker SA, Tekchandani L, Thomas M, et al. Newly diagnosed breast cancer: comparison of contrast-enhanced spectral mammography and breast MR imaging in the evaluation of extent of disease. Radiology. 2017;285:389-400. https://doi.org/10. 1148/radiol.2017161592.

61. Jochelson MS, Pinker K, Dershaw DD, et al. Comparison of screening CEDM and MRI for women at increased risk for breast cancer: a pilot study. Eur J Radiol. 2017;97:37-43. https://doi. org/10.1016/j.ejrad.2017.10.001.

62. • Sung JS, Lebron L, Keating D, et al. Performance of dualenergy contrast-enhanced digital mammography for screening women at increased risk of breast cancer. Radiology. 2019;293:81-8. https://doi.org/10.1148/radiol.2019182660. This study have showed that contrast-enhanced digital mammography is a promising technique for screening women with higher-thanaverage risk for breast cancer.

63. Li L, Roth R, Germaine P, et al. Contrast-enhanced spectral mammography (CESM) versus breast magnetic resonance imaging (MRI): a retrospective comparison in 66 breast lesions. Diagn Interv Imaging. 2017;98:113-23. https://doi.org/10.1016/j. diii.2016.08.013.

64. Patel BK, Gray RJ, Pockaj BA. Potential cost savings of contrastenhanced digital mammography. Am J Roentgenol. 2017;208:W231-7. https://doi.org/10.2214/AJR.16.17239.

65. Hobbs MM, Taylor DB, Buzynski S, Peake RE. Contrast-enhanced spectral mammography (CESM) and contrast enhanced MRI (CEMRI): patient preferences and tolerance. J Med Imaging Radiat Oncol. 2015;59:300-5. https://doi.org/10.1111/1754-9485. 12296.

66. Phillips J, Miller MM, Mehta TS, et al. Contrast-enhanced spectral mammography (CESM) versus MRI in the high-risk screening setting: patient preferences and attitudes. Clin Imaging. 2017;42:193-7. https://doi.org/10.1016/j.clinimag.2016.12.011.

67. Gulani V, Calamante F, Shellock FG, et al. Gadolinium deposition in the brain: summary of evidence and recommendations. Lancet Neurol. 2017;16:564-70. https://doi.org/10.1016/S14744422(17)30158-8.
68. Mainiero MB, Moy L, Baron P, et al. ACR appropriateness criteria ${ }^{\circledR}$ breast cancer screening. J Am Coll Radiol. 2017;14:S383-90. https://doi.org/10.1016/j.jacr.2017.08.044.

69. Hogan MP, Amir T, Sevilimedu V, et al. Contrast-enhanced digital mammography screening for intermediate-risk women with a history of lobular neoplasia. Am J Roentgenol. 2021;216:1486-91. https://doi.org/10.2214/AJR.20.23480.

70. Suter MB, Pesapane F, Agazzi GM, et al. Diagnostic accuracy of contrast-enhanced spectral mammography for breast lesions: a systematic review and meta-analysis. Breast. 2020;53:8-17. https://doi.org/10.1016/j.breast.2020.06.005.

71. Patel BK, Garza SA, Eversman S, et al. Assessing tumor extent on contrast-enhanced spectral mammography versus full-field digital mammography and ultrasound. Clin Imaging. 2017;46:78-84. https://doi.org/10.1016/j.clinimag.2017.07.001.

72. ElSaid NAES, Mahmoud HGM, Salama A, et al. Role of contrast enhanced spectral mammography in predicting pathological response of locally advanced breast cancer post neo-adjuvant chemotherapy. Egypt J Radiol Nucl Med. 2017;48:519-27. https://doi.org/10.1016/j.ejrnm.2017.03.022.

73. Iotti V, Ravaioli S, Vacondio R, et al. Contrast-enhanced spectral mammography in neoadjuvant chemotherapy monitoring: a comparison with breast magnetic resonance imaging. Breast Cancer Res. 2017. https://doi.org/10.1186/s13058-017-0899-1.

74. Patel BK, Hilal T, Covington M, et al. Contrast-enhanced spectral mammography is comparable to MRI in the assessment of residual breast cancer following neoadjuvant systemic therapy. Ann Surg Oncol. 2018;25:1350-6. https://doi.org/10.1245/ s10434-018-6413-x.

75. Sener SF, Sargent RE, Lee C, et al. MRI does not predict pathologic complete response after neoadjuvant chemotherapy for breast cancer. J Surg Oncol. 2019;120:903-10. https://doi.org/ $10.1002 /$ jso. 25663.

76. Luk L, Steinman J, Newhouse JH. Intravenous contrast-induced nephropathy - the rise and fall of a threatening idea. Adv Chronic Kidney Dis. 2017;24:169-75. https://doi.org/10.1053/j.ackd. 2017.03.001.

77. Lewin J. Comparison of contrast-enhanced mammography and contrast-enhanced breast MR imaging. Magn Reson Imaging Clin N Am. 2017;26:259-63. https://doi.org/10.1016/j.mric.2017.12. 005.

78. Hendrick RE. Radiation doses and cancer risks from breast imaging studies. Radiology. 2010;257:246-53. https://doi.org/10. 1148/radiol.10100570.

79. Covington MF, Pizzitola VJ, Lorans R, et al. The future of contrast-enhanced mammography. Am J Roentgenol. 2018;210:292-300. https://doi.org/10.2214/AJR.17.18749.

80. Dromain C, Thibault F, Diekmann F, et al. Dual-energy contrastenhanced digital mammography: initial clinical results of a multireader, multicase study. Breast Cancer Res. 2012. https:// doi.org/10.1186/bcr3210.

Publisher's Note Springer Nature remains neutral with regard to jurisdictional claims in published maps and institutional affiliations. 